\title{
Optimal Fleet Deployment Strategy: Model the Effect of Shared E-Bikes on Bike-Sharing System
}

\author{
Siying Zhu \\ School of Civil and Environmental Engineering, Nanyang Technological University, Singapore \\ Correspondence should be addressed to Siying Zhu; siying001@e.ntu.edu.sg
}

Received 13 December 2020; Revised 23 January 2021; Accepted 18 February 2021; Published 26 February 2021

Academic Editor: Chung-Cheng Lu

Copyright $\odot 2021$ Siying Zhu. This is an open access article distributed under the Creative Commons Attribution License, which permits unrestricted use, distribution, and reproduction in any medium, provided the original work is properly cited.

Following the bike-sharing system, the shared e-bike becomes increasingly popular due to the advantage in speed, trip distance, and so forth. However, limited research has investigated the impact of the introduction of shared e-bikes on the existing bike-sharing systems. This paper aims to study the effect of shared e-bikes on the traditional bike-sharing system and determine the optimal fleet deployment strategy under a bimodal transportation system. A stochastic multiperiod optimisation model is formulated to capture the demand uncertainty of travelers. The branch-and-bound algorithm is applied to solve problem. A 15-station numerical example is applied to examine the validity of the model and the effectiveness of the solution algorithm. The performance of integrated e-bike and bike-sharing system has been compared with the traditional bike-sharing system. The impacts of the charging efficiency, fleet size, and pricing strategy of e-bike-sharing system on the traditional bike-sharing system have been examined.

\section{Introduction}

In recent years, bike-sharing systems (BSSs) have significantly grown in prevalence and popularity in the urban environment, providing a healthy, low-cost, and environmentally friendly mode of transportation. With years of development, operational challenges have been overcome by BSS operators to offer fully automated, secure, and cost-effective bike-sharing service due to the technological advances such as the improvement in mobile technology, electronic payment, and GPS-based devices [1].

Following BSS, the shared electric bicycle or shared e-bike is another type of vehicle catalysed by the era of shared operations [2]. Traditional bicycles have been replaced with e-bikes in many cities due to traditional bicycle's disadvantage in speed, trip distance, and vulnerability under adverse weather condition, uneven terrain, and so forth $[3,4]$. However, e-bikes are more expensive than the traditional bicycles in terms of both capital cost and operational cost. Infrastructure such as charging stations is also required to be constructed to support the operation of e-bike-sharing system (EBSS). Pilot EBSSs have been deployed all over the world, such as North America [5, 6], Europe [7-10], and Asia [11, 12].

Despite recognising the potential costs and benefits of shared e-bikes, limited attention has been paid to analysing the impact of the introduction of shared e-bikes on the existing BSSs. This paper aims to analyse the effect of shared e-bikes on the traditional BSSs under a bimodal transportation system, with four main contributions as follows: (1) The optimal fleet deployment strategy of EBSS is determined for an integrated system with both e-bikes and traditional bikes. A bimodal user network equilibrium is formulated to address the impact of the introduction of shared e-bikes on bicyclists' mode choice behaviour. (2) Stochastic travel demand is considered to address the demand uncertainty of commuters. (3) The performances of integrated e-bike and bike-sharing system and the traditional BSS are compared. The impact of the characteristics of EBSS, including the fleet size, charging efficiency, and pricing strategy has been investigated. The evaluation of EBSS's impact on the existing BSSs is fundamental to determine the feasibility of EBSS, which can assist service provider and city planners design and manage the operation of EBSS effectively and efficiently. (4) 
The branch-and-bound algorithm is applied to solve the problem and a numerical example is utilised to test the validity of the model and the effectiveness of the solution algorithm.

\section{Related Work}

2.1. E-Bike. As e-bikes are one of the fast-growing modes of transportation in the market, many studies have been dedicated to e-bikes. Fishman and Cherry [13] provided a comprehensive review on e-bike-related literature, whose focus is transport instead of recreational e-bike research. E-bikes were shown to reduce the fatigue level [14-17] and increase the cyclist's mode satisfaction level [18]. It was also found that e-bikes can increase the ridership or travel distance in comparison with traditional bikes $[4,15,19,20]$. Moreover, there were also papers which addressed the health impacts [21, 22], environmental impacts [22-24], and the safety issues of e-bikes [23, 25-29].

2.2. E-Bike-Sharing System. As e-bikes are more expensive than traditional bikes, e-bike sharing is a potential solution to the price barrier. Cherry et al. [30] outlined the basic system requirements and operational concepts of EBSS. Manzoni et al. [31] presented a BSS architecture with the integration of e-bikes. Langford et al. [5] presented an overview of the first EBSS in North America, which offered a sustainable mode of transportation for the students. Campbell et al. [32] employed stated preference survey to identify the factors influencing the choice of shared bikes and shared e-bikes. He et al. [6] utilised the historical trip data to identify the factors that affect EBSS ridership. Ji et al. [33] simulated the user demand and system availability of EBSS based on a pilot e-bike-sharing project. Li et al. [34] proposed a method for sharing e-bikes trajectory data cleansing. To optimise the EBSS design systematically, several studies have formulated optimisation models. Zhong et al. [2] investigated the optimal deployment strategy of static charging piles for EBSS. Chen et al. [3], Hu et al. [35], and Martinez et al. [36] determined the optimal allocation strategy of e-bike-sharing stations. However, the influence of shared e-bikes on the existing BSSs has not been analysed systematically with mathematic models in previous research.

2.3. Fleet Deployment Strategy. Although the fleet deployment strategy for EBSS has drawn less attention, the determination of the optimal inventory level of traditional bikes at each bike station or fleet deployment strategy has been widely investigated. Sayarshad et al. [37] formulated a multiperiod optimisation model to determine the minimum bike fleet size for maximising the total benefit to the bike-sharing service provider. Lin et al. [38] formulated a hub location inventory model and a heuristic solution method to address the BSS design problem with bicycle stock considerations. Lu [39] determined the optimal number of bicycles deployed at each bicycle station for multiple time periods in a short-term planning horizon, which aims to minimise the total system costs. Yan et al. [40] and Zhu [41] optimised the number of bikes allocated to each bike station under stochastic demand. Caggiani et al. [42] determined the allocation strategy of racks and bicycles to maximise the level of service such that users are not forced to use other stations or turn to other modes of transportation.

\section{Methodology}

3.1. Notations and Assumptions. Notations of sets, indices, and variables used in the integrated bike and e-bike-sharing system fleet deployment model are summarised in Table 1, respectively. Moreover, assumptions are made for simplicity:

(1) This study focuses on trips in BSS/EBSS, wherein two modes of transportation, traditional bike and e-bike, are considered. Each station is able to accommodate both bikes and e-bikes. In other words, each station can be considered as both shared bike station and shared e-bike station.

(2) Commuters can only use one mode of transportation between each pair of bike/e-bike stations.

(3) The travel time by bike and e-bike is not affected by the traffic volume. In other words, the travel speed of bicyclist and e-bicyclist on each route is fixed.

(4) The repositioning process of bikes is only carried out at the end of the planning horizon; that is, static rebalancing strategy is applied.

(5) A set of discrete scenarios with given probability distribution are applied to represent the uncertain demand distribution at various bike/e-bike stations, which are assumed to be known a priori. The probability distribution of demand can be estimated and sampled from bike/e-bike-sharing operators' historical trip data.

(6) The duration of each trip is less than the length of planning period.

(7) The charging time is linear with regard to the amount of electricity to be charged, as the charging speed is assumed to be constant.

\subsection{Generalised Travel Cost}

3.2.1. Traditional Bike. The generalised trip cost of traditional bike is comprised of three parts, namely, the cycling time, the rental fee, and the comfort cost:

$$
t_{i j}^{g}=t_{i j}^{c}+t_{i j}^{r}+t_{i j}^{f}, \quad \forall i, j .
$$

The cycling time $t_{i j}^{c}$ of traditional bike between station $i$ and station $j$ is calculated as

$$
t_{i j}^{c}=\frac{d_{i j}}{s}, \quad \forall i, j,
$$

where $d_{i j}$ denotes the distance between stations $i$ and $j$ and $s$ denotes the average speed of traditional bike.

The rental fee component of generalised trip cost $t_{i j}^{r}$ (in hours) of traditional bike between stations $i$ and $j$ is calculated as 
TABle 1: Notations.

\begin{tabular}{|c|c|}
\hline$N$ & The set of bike stations \\
\hline$\widehat{\widehat{N}}$ & The set of e-bike stations, $\widehat{N} \subseteq N$ \\
\hline$i, j$ & The indices of nodes in $N, i, j \in N$ \\
\hline$\Omega$ & The support of the demand distribution \\
\hline$e$ & Subscript associated with variables for certain demand realisation, $e \in \Omega$ \\
\hline E & The set of demand scenarios, $E=\{1,2, \ldots,|E|\}$ \\
\hline$p_{e}$ & Probability of demand scenario $e$ \\
\hline$T$ & The set of periods in the planning horizon \\
\hline$t$ & The index of any given time period in the planning horizon \\
\hline$l(t)$ & Length of planning time period $t$ \\
\hline$t_{i j}^{g}$ & The generalised trip cost of traditional bike between stations $i$ and $j$ \\
\hline$t_{i j}^{c}$ & The cycling time of traditional bike between stations $i$ and $j$ \\
\hline$t_{i j}^{r}$ & The rental fee component of generalised trip cost of traditional bike between stations $i$ and $j$ \\
\hline$t_{i j}^{f}$ & The comfort cost of riding traditional bike between stations $i$ and $j$ \\
\hline$\widehat{t}_{i j}$ & The generalised trip cost of e-bikes between stations $i$ and $j$ \\
\hline$\widehat{t}_{i j}^{c}$ & The cycling time of e-bike between stations $i$ and $j$ \\
\hline$\widehat{t}_{i j}^{i j}$ & The rental fee component of generalised trip cost of e-bike between stations $i$ and $j$ \\
\hline$d_{i j}$ & Distance between station $i$ and station $j$ \\
\hline$s$ & Average speed of traditional bike \\
\hline$\widehat{s}$ & Average speed of e-bike \\
\hline $\bar{\tau}$ & Average charging time of each e-bike arrived at e-bike station after rental, in order to reach sufficient state of charge (SOC) \\
\hline$e^{s}$ & The minimum sufficient SOC \\
\hline$e^{o}$ & The initial average SOC \\
\hline$\omega_{i}$ & The charging speed at e-bike station $i$ \\
\hline$\eta$ & The value of time of commuters \\
\hline$\eta^{f}$ & The value of fatigue of bicyclists \\
\hline$r_{i j}$ & Monetary unit rental fee per utilised bike sent from station $i$ to station $j$ \\
\hline$\hat{r}_{i j}$ & Monetary unit rental fee per utilised e-bike sent from station $i$ to station $j$ \\
\hline$c_{i}^{h}$ & Holding cost per empty bike at station $i$ \\
\hline$\widehat{c}_{i}^{h}$ & Holding cost per empty e-bike at station $i$ \\
\hline$c^{\text {elec }}$ & Unit electricity price \\
\hline$\beta$ & Energy consumption rate of e-bike \\
\hline$m_{i}^{r}$ & Number of bike racks of station $i$, that is, capacity of station $i$ for traditional bikes \\
\hline$m_{i}^{c}$ & Number of charging piles of station $i$, that is, capacity of station $i$ for e-bikes \\
\hline$\underline{u}$ & Minimum required bike fleet utilisation rate (trips per bike per day) \\
\hline$\underline{\widehat{u}}$ & Minimum required e-bike fleet utilisation rate (trips per bike per day) \\
\hline $\bar{u}$ & Total bike/e-bike fleet utilisation rate (trips per bike per day) \\
\hline$B$ & Fleet size of bikes to be deployed \\
\hline$\widehat{B}$ & Fleet size of e-bikes to be deployed \\
\hline$Q_{i j}^{e}(t)$ & Total demand for shared bike/e-bike transportation service from station $i$ to station $j$ in time period $t$ for demand realisation $e$ \\
\hline$q_{i j}^{e j}(t)$ & Demand for shared bike transportation service from station $i$ to station $j$ in time period $t$ for demand realisation $e$ \\
\hline$\widehat{q}_{i j}^{e}(t)$ & Demand for shared e-bike transportation service from station $i$ to station $j$ in time period $t$ for demand realisation $e$ \\
\hline$x_{i j}^{e}(t)$ & The number of rented bikes dispatched from station $i$ to station $j$ in time period $t$ in demand scenario $e$ \\
\hline$v_{i}^{e}(t)$ & The number of bikes presented at station $i$ at the beginning of the period $t$ in demand scenario $e$ \\
\hline$\hat{x}_{i j}^{e}(t)$ & The number of rented e-bikes dispatched from station $i$ to station $j$ in time period $t$ in demand scenario $e$ \\
\hline$\widehat{v}_{i}^{e}(t)$ & The number of e-bikes presented at station $i$ at the beginning of the period $t$ in demand scenario $e$ \\
\hline$\theta$ & A positive parameter in modal split function \\
\hline
\end{tabular}

$$
t_{i j}^{r}=\frac{t_{i j}^{c} \cdot r_{i j}}{\eta}, \quad \forall i, j,
$$

where $\eta$ is the value of time of commuters. The parameter $\eta$ denotes the amount of money that a traveler would like to pay in order to save one unit of time. The value of $\eta$ depends on traveler's preferences, which should be adjusted with trip survey data for real-world application. $r_{i j}$ denotes the monetary unit rental fee per utilised bike sent from station $i$ to station $j$, which is assumed to be constant with unit in \$/hour.
The comfort cost of riding traditional bike $t_{i j}^{f}$ between station $i$ and station $j$ is calculated based on the riding fatigue function in Li et al.'s work [43] to reflect the degree of muscle pain and physical fatigue of bicyclists due to the bicycle ride, where the fatigue level of bicyclist is related to the riding time.

$$
t_{i j}^{f}=\frac{\alpha_{1} \cdot t_{i j}^{c}+\alpha_{2} \cdot t_{i j}^{c 2}}{\eta^{f}},
$$

where $\alpha_{1}$ and $\alpha_{2}$ are positive parameters, which can be calibrated with survey data; $\eta^{f}$ is the value of fatigue of 
bicyclists, representing the amount of disutility perception in terms of the comfort cost that a traveler would like to pay in order to save one unit of time. The value of $\eta^{f}$ also depends on traveler's preferences, which should be calibrated with trip survey data for real-world application. The comfort cost of e-bike is assumed to be negligible in this paper, as the electric assistance can reduce the fatigue barrier. Please note that, for real-world application, the values of $\alpha_{1}$ and $\alpha_{2}$ should be calibrated with survey data, as these parameters are related to bike lane performance and bicyclists' physical condition. The fixed parameter values used in this paper are only for numerical testing purpose.

3.2.2. E-Bike. The generalised trip cost of e-bikes contains the cycling time and the rental fee.

$$
\widehat{t}_{i j}=\widehat{t}_{i j}^{c}+\widehat{t}_{i j}^{r}, \quad \forall i, j \text {. }
$$

The cycling time $\widehat{t}_{i j}^{c}$ of e-bike between stations $i$ and $j$ is calculated as

$$
\widehat{t}_{i j}^{c}=\frac{d_{i j}}{\widehat{s}}, \quad \forall i, j .
$$

where $\widehat{s}$ denotes the average speed of e-bike.

The rental fee component of generalised trip cost $\hat{t}_{i j}^{r}$ (in hours) of e-bike between stations $i$ and $j$ is calculated as

$$
\widehat{t}_{i j}^{r}=\frac{\widehat{t}_{i j}^{c} \cdot \widehat{r}_{i j}}{\eta}, \quad \forall i, j,
$$

where $\widehat{r}_{i j}$ denotes the monetary unit rental fee per utilised e-bike sent from station $i$ to station $j$.

3.3. Bilevel Model. A bilevel model is formulated to determine the optimal fleet deployment strategy and address the effect of shared e-bikes on the bike-sharing system. The upper level aims to determine the fleet deployment strategy to optimise the earned profit of the overall system, which is affected by the bike/e-bike flow variables. On the other hand, the flow variables should be derived from the lower-level model, which addresses the multimodal user equilibrium condition. In other words, the lower-level model is embedded or nested within the upper-level model. More detailed explanations are provided in the following sections.

3.3.1. Upper-Level Model. The problem formulation principally determines the optimal fleet deployment strategy to each bike/e-bike station, with the objective to maximise the expectation of the earned profit for the bike/e-bike-sharing operator. The first line in the objective function (equation (8)) represents the total revenue from rented bikes/e-bikes. The second line represents the total holding cost of bikes/e-bikes at stations. The third line represents the charging cost of e-bikes. The focus of this paper is to investigate the effect of e-bikes on existing BSS with traditional shared bikes only.

$$
\begin{aligned}
Z= & E\left[\sum_{i} \sum_{j} \sum_{t} r_{i j} \cdot x_{i j}^{e}(t)+\sum_{i} \sum_{j} \sum_{t} \widehat{r}_{i j} \cdot \widehat{x}_{i j}^{e}(t)\right. \\
& -\sum_{i} \sum_{t}\left(c_{i}^{h} \cdot l(t) \cdot\left(v_{i}^{e}(t)-\sum_{j} x_{i j}^{e}(t)\right)\right)-\sum_{i} \sum_{t}\left(\widehat{c}_{i}^{h} \cdot l(t) \cdot\left(\widehat{v}_{i}^{e}(t)-\sum_{j} \widehat{x}_{i j}^{e}(t)\right)\right) \\
& \left.-\sum_{i} \sum_{j} \sum_{t} c^{\text {elec }} \cdot \beta \cdot d_{i j} \cdot \hat{x}_{i j}^{e}(t)\right],
\end{aligned}
$$

where $c^{h}$ and $\widehat{c}^{h}$ denote the unit holding costs for traditional bike and e-bike, respectively. The bikes/e-bikes that are not rented can incur a holding cost, that is, the cost of holding unused bikes/e-bikes at a station for a time interval such as 1 hour when the BSS/EBSS is in operation. The unit holding cost should be derived by dividing the weekly system management cost by the product of station number, operating hours, and fleet size [39]. In this paper, similar to Sayarshad et al. [37], the cost of holding a bike at each station is calculated for each time period $t$ (as shown in equation (8)). If a bicycle is kept stagnant/idle at a particular station $i$ for all the time periods except for the last one, the holding cost for that particular bicycle should be calculated for all the past accumulative time periods, as the idle bicycle contributes to $v_{i}^{e}(t)$ at station $i$ but does not contribute to $x_{i j}^{e}(t)$, except for the last time period $t$. $c^{\text {elec }}$ denotes the unit electricity price; $\beta$ denotes the energy consumption rate of e-bike; $d_{i j}$ denotes the distance between stations $i$ and $j$. Note that, similar to Yan et al. [40], we do not consider supply cost like bike purchasing and depreciation cost for bikes/e-bikes as the fleet is assumed to be already given. $e$ denotes the subscript associated with variables for certain demand realisation.

The constraints of the model are listed as follows: 


$$
\begin{aligned}
& v_{i}^{e}(t)=v_{i}^{e}(t-1)+\sum_{j} x_{j i}^{e}(t-1)-\sum_{j} x_{i j}^{e}(t-1), \quad \forall i, j, t, e, \\
& \widehat{v}_{i}^{e}(t)=\widehat{v}_{i}^{e}(t-1)+\sum_{j} \hat{x}_{j i}^{e}(t-\lceil\bar{\tau} /|t|\rceil)-\sum_{j} \hat{x}_{i j}^{e}(t-1), \quad \forall i, j, t, e, \\
& \underset{e}{E}\left[\sum_{i} \sum_{j} \sum_{t} x_{i j}^{e}(t)\right] \geq \underline{u} \cdot \underset{e}{E}\left[\sum_{i} v_{i}^{e}(1)\right], \quad \forall i, t \\
& \underset{e}{E}\left[\sum_{i} \sum_{j} \sum_{t} \hat{x}_{i j}^{e}(t)\right] \geq \underline{\widehat{u}} \cdot \underset{e}{E}\left[\sum_{i} \widehat{v}_{i}^{e}(1)\right], \quad \forall i, t, \\
& v_{i}^{1}(1)=v_{i}^{2}(1)=\cdots=v_{i}^{|E|}(1), \quad \forall i, \\
& \widehat{v}_{i}^{1}(1)=\widehat{v}_{i}^{2}(1)=\cdots=\widehat{v}_{i}^{|E|}(1), \quad \forall i, \\
& \sum_{j} x_{i j}^{e}(t) \leq v_{i}^{e}(t), \quad \forall i, j, t, e, \\
& \sum_{j} \widehat{x}_{i j}^{e}(t) \leq \widehat{v}_{i}^{e}(t), \quad \forall i, j, t, e, \\
& v_{i}^{e}(t) \leq m_{i}^{r}, \quad \forall i, t, e, \\
& \widehat{v}_{i}^{e}(t) \leq m_{i}^{c}, \quad \forall i, t, e, \\
& \sum_{i} v_{i}^{1}(1)=\sum_{i} v_{i}^{2}(1)=\cdots=\sum_{i} v_{i}^{|E|}(1)=B, \quad \forall i, \\
& \sum_{i} \widehat{v}_{i}^{1}(1)=\sum_{i} \widehat{v}_{i}^{2}(1)=\cdots=\sum_{i} \widehat{v}_{i}^{|E|}(1)=\widehat{B}, \quad \forall i, \\
& x_{i j}^{e}(t), v_{i}^{e}(t), \widehat{x}_{i j}^{e}(t), \widehat{v}_{i}^{e}(t) \in \mathbb{N}^{0}, \quad \forall i, j, t, e .
\end{aligned}
$$

Equations (9) and (10) represent flow conservation constraints at each bike/e-bike station in each time period for traditional bikes and e-bikes, respectively. For EBSS, the occupied charging pile changes to unoccupied when the e-bike is rented. On the other hand, the unoccupied charging pile turns into occupied when the e-bike is returned [3]. $\bar{\tau}$ denotes the average charging time of each e-bike arriving at e-bike station after rental, in order to reach sufficient state of charge (SOC):

$$
\bar{\tau}=\frac{e^{s}-e^{o}}{\omega_{i}},
$$

where $e^{s}$ denotes the minimum sufficient SOC, $e^{o}$ denotes the initial average SOC, and $\omega_{i}$ denotes the charging speed at e-bike station $i$. Following the common practice, the shared e-bike battery should be charged-discharged between $20 \%$ and $80 \%$ of the battery capacity.

Equations (11) and (12) represent that the bike and e-bike fleet utilisation rate has to be greater than the minimum required values $\underline{u}$ and $\underline{\hat{u}}$ to avoid oversupply in the market. Equations (13) and (14) ensure that the same fleet deployment plan is applied at the initial period of planning horizon for different demand realisations. Constraints (15) and (16) are balancing constraints for the number of bikes and e-bikes at the beginning of each period $t$. Constraint (17) ensures that the number of bikes at each station can never exceed the capacity of bike station; constraint (18) ensures that the number of e-bikes at each e-bike station cannot exceed the total number of charging piles. Equations (19) and (20) are fleet size constraints to ensure that the total fleet size in each demand scenario is the same, and the fleet size constraint can also serve as a budget constraint. Constraint (21) defines the domain of design variables, which are nonnegative integers.

3.3.2. Lower-Level Model. To derive the flow variables used in the upper level of the problem, multimodal user equilibrium (MUE) model is formulated in the lower level of the model to address the traffic assignment between e-bikes and traditional bikes. Under stochastic travel demand, it is not 
sensible to find the MUE flow pattern while expecting that the travel cost remains the same. Instead, we aim to derive the MUE traveler flow pattern for each demand realisation. The MUE condition include two aspects: first, all utilised paths of the same travel mode have the same travel cost between each OD pair and the travel costs of all unutilised paths are not lower than the travel costs of any utilised paths for the same travel mode; second, commuters' mode choice decisions are based on the multinomial logit model [44].

Based on assumption (3), for the first MUE condition, the travel time between each pair of stations $i$ and $j$ is fixed, which is equal to the travel time on the shortest path. Constraints (23) and (24) represent that the actual number of bicycles and e-bikes dispatched between two stations cannot exceed the demand for bikes and e-bikes, respectively. Constraint (25) ensures that the total travel demand between each two stations is equal to the summation of demand for shared bikes and e-bikes:

$$
\begin{array}{r}
x_{i j}^{e}(t) \leq q_{i j}^{e}(t), \quad \forall i, j, t, e, \\
\hat{x}_{i j}^{e}(t) \leq \hat{q}_{i j}^{e}(t), \quad \forall i, j, t, e, \\
q_{i j}^{e}(t)+\hat{q}_{i j}^{e}(t)=Q_{i j}^{e}(t), \quad \forall i, j, t, e .
\end{array}
$$

For the second MUE condition, the modal split is based on the logit function, which is formulated as follows:

$$
q_{i j}^{e}(t)=\frac{Q_{i j}^{e}(t)}{1+\exp \left[\theta\left(t_{i j}^{g}-\widehat{t}_{i j}^{g}\right)\right]}, \quad \forall i, j, t, e,
$$

where $t_{i j}^{g}$ and $\hat{t}_{i j}^{g}$ denote the generalised travel cost between stations $i$ and $j$ by traditional bike and e-bike, respectively, and $\theta$ is a positive parameter. Parameter $\theta$ denotes the modal split parameter, which affects the estimation of the proportion of trip-makers who are likely to use each mode (traditional bike/e-bike) to travel between each pair of stations. The value of $\theta$ should be calibrated with real-world trip survey data. For numerical testing purpose, a constant value of $\theta$ is assumed in this paper.

\section{Solution Algorithm}

The decision variables for the shared bike/e-bike fleet deployment optimisation model are all integers, representing the number of bikes/e-bikes. It is unfeasible to enumerate all the candidate fleet deployment plans to derive the optimal strategy due to the huge computational effort. The branch-and-bound algorithm (as shown in Algorithm 1) is applied in this paper to solve the fleet deployment optimisation problem with the discrete feature of bike/e-bike flow. A sequence of subproblems are constructed by the algorithm, with the objective to converge to an optimal solution to the integer linear programming model. The subproblems contain a set of upper bounds and lower bounds where the initial upper bound is the solution to the relaxed problem without integer constraints and the initial lower bound is any feasible solution derived with heuristics.
Branching from the root node, new subproblems are constructed by choosing various variables to split based on branching rules. The branching rule for choosing fractional variable is based on the reliability, for which more details can be found in [46]. Generally, the branch-and-bound algorithm creates subproblems for analysis and the ones that cannot improve the lower bound of the model are eliminated from the searching region. The searching process is terminated when the difference between the lower bound and the upper bound is within a tolerance value or when the number of explored nodes has reached the preset limit.

\section{Numerical Example}

5.1. Input Data. In this section, most of the parameter values are approximated according to related literature $[3,33,37,40,43]$. The planning horizon is set to 12 hours, and static rebalancing method is applied. The bike and e-bike rental fees $r_{i j}$ and $\widehat{r}_{i j}$ are set as 1 and 3 USD/hour, respectively. The holding costs per empty traditional bike $c_{i}^{h}$ and e-bike $\widehat{c}_{i}^{h}$ are set as $0.05 \mathrm{USD} /$ hour and $0.07 \mathrm{USD} / \mathrm{hour}$, respectively. $\alpha_{1}$ and $\alpha_{2}$ are set as $0.2 \$ /$ hour and $0.1 \$ /$ hour $^{2}$, respectively. $\eta$ and $\eta^{f}$ are set as $10 \$$ /hour and (2/3) \$/hour, respectively. $\theta$ is set as 10 . The unit electricity price $c^{\text {elec }}$ is set as $0.2 \mathrm{USD} / \mathrm{kw} \cdot \mathrm{h}$; the energy consumption rate of e-bike $\beta$ is set as $2.1 \mathrm{kw} \cdot \mathrm{h} / 100 \mathrm{~km}$. According to Cherry et al. [30], the recharge time of e-bikes in EBSS normally ranges between 4 and 6 hours, and the time could be reduced to 30 minutes with the help of more advanced battery and recharging technology. The average charging time $\bar{\tau}$ is firstly set as 0.5 hours. Other charging times will also be tested out in the following sections. 15 stations are considered, where each of them is able to accommodate both bikes and e-bikes. The demand level is assumed to follow normal distribution, which can be simulated with Monte Carlo sampling method: the mean demand between each pair of stations for each 2-hour interval is set as random values between 20 and 50 trips, the standard deviation of demand in time interval is set as 5 , and the sample size is set as 5 . The average speeds of riding traditional bike $s$ and e-bike $\widehat{s}$ are set as $11 \mathrm{~km} / \mathrm{h}$ and $15 \mathrm{~km} / \mathrm{h}$, respectively. The distances between each pair of station are set as some random number between $1 \mathrm{~km}$ and $4 \mathrm{~km}$. The maximum capacity of each bike station $m_{i}^{r}$ and e-bike station $m_{i}^{c}$ is set as 50 in both. The minimum required bike fleet utilisation rates $\underline{u}$ and $\underline{\hat{u}}$ are both set as 5 . The total fleet sizes of traditional bike $B$ and e-bike $\widehat{B}$ are both set as 500 . For the solution method, $\epsilon$ is set as $10^{-5}$.

5.2. Fleet Deployment Strategy. The optimal fleet deployment strategy at each station at the beginning of the time period is determined and demonstrated in this section. Three systems are considered:

(i) System 1: BSS and EBSS coexist in an integrated system, where the capacities of both BSS and EBSS are the same and total fleet size of bikes and e-bikes are equal.

(ii) System 2: only BSS exists in the system. The capacity of each station is the sum of the capacities of BSS 
Step 0: initialization and BSS/EBSS loading

(a) Set up the parameters;

(b) Load the integrated bike and e-bike sharing system;

(c) The objective is to find the fleet deployment solution $Y$ that maximises the earned profit $Z(Y)$ in equation (8);

(d) Let $k=0$ be the number of iteration.

Step 1: solve relaxed noninteger problem

The relaxed problem is solved without integer constraints based on dual-simplex algorithm [45] for linear programming. The results $Y_{l p}$ correspond to the first upper bound: $Z(Y) \leq Z\left(Y_{l p}\right) U B$.

Step 2: Find feasible solutions with heuristics

Heuristics is applied to generate a feasible point $Y^{k}$, such that the lower bound of the fleet deployment optimisation model can be determined based on $Y^{k}: Z(Y) \geq Z\left(Y^{k}\right)=L B^{k}$.

Step 3: iteration

The algorithm is terminated when meeting the stopping criteria as follows:

(a) The difference between the lower bound and upper bound is less than the tolerance value, i.e. $U B-L B^{k} \leq \epsilon$;

(b) The number of nodes explored exceeds the maximum number of nodes.

Otherwise, repeat steps 4-5.

Step 4: branching

Noninteger variable $w$ with value $\hat{y}_{w}$ is chosen as the fractional variable to split;

$k=k+1$;

Two subproblems are generated by adding constraints for the variable $w$ as $y_{w} \leq\left\lfloor\hat{y}_{w}\right\rfloor$ and $y_{w} \geq\left\lceil\hat{y}_{w}\right\rceil$ respectively;

Based on step 1 and step 2, determine the lower bounds $\left(L B_{1}^{k}, L B_{2}^{k}\right)$ of the two subproblems with the corresponding decision variable vectors $Y_{1}^{k}$ and $Y_{2}^{k}$ respectively.

Step 5: update

$L B^{k}=\max \left(L B^{k-1}, L B_{1}^{k}, L B_{2}^{k}\right)$;

if $L B^{k}=L B^{k-1}$ then

The corresponding region is eliminated from consideration as no branching is possible;

$Y^{k}=Y^{k-1}$

else if $L B^{k}=L B_{1}^{k}$ then

Additional constraint $y_{w} \leq\left\lfloor\hat{y}_{w}\right\rfloor$ is added to the searching region;

$Y^{k}=Y_{1}^{k}$

Proceed to step 3;

else

Additional constraint $y_{w} \geq\left[\hat{y}_{w}\right]$ is added to the searching region;

$Y^{k}=Y_{2}^{k}$

Proceed to step 3.

end

Algorithm 1: Branch-and-bound algorithm.

and EBSS in system 1 . The total fleet size of traditional bikes is equal to the sum of the fleet size of bikes and e-bikes in system 1.

(iii) System 3: only BSS exists in the system. The capacity of each station is the same as the capacity of BSS system 1 . The total fleet size of traditional bikes is equal to the fleet size of bikes in system 1 .

The numerical example is tested on a personal computer with Intel Core i5 CPU $2.3 \mathrm{GHz}, 8 \mathrm{~GB}$ RAM, and MacOS 10.14. The running time for system 1 with full set of constraints utilised is about 15 seconds, and the running time is around 7 seconds for the other two systems. For the three systems mentioned above, the optimal bike/e-bike fleet deployment strategy at each bike/e-bike station at the beginning of the planning period is summarised in Table 2. Please note that there is no e-bike in systems 2 and 3, such that the lower-level model should be revised as equations (27)-(28) as below, representing that cyclists are only allowed to rent traditional bikes:

$$
\begin{aligned}
& x_{i j}^{e}(t) \leq q_{i j}^{e}(t), \quad \forall i, j, t, e, \\
& q_{i j}^{e}(t)=Q_{i j}^{e}(t), \quad \forall i, j, t, e .
\end{aligned}
$$

The fleet deployment strategies in Table 2 also show some interesting observations: For example, for station 7, in systems 1 and 2, the optimal number of bikes/e-bikes deployed to station 7 at the beginning of the planning horizon is exactly equal to the capacity. On the other hand, for system 3, at the beginning of the planning horizon, the number of bikes deployed to the station is not as full. This is because the optimal fleet deployment strategy is determined based on a dynamic modelling process, which aims to optimise the earned profit of the entire rental system within the whole planning horizon. Thereby, the three rental systems with various types of bicycles and capacity constraints can lead to different types of fleet deployment strategies (e.g., the number of bikes deployed at station 7) at the beginning of the planning horizon. It can also be observed that, except for station 15, the number of bikes deployed in system 2 at the 
TABLE 2: Fleet deployment strategy.

\begin{tabular}{|c|c|c|c|c|c|c|c|c|c|c|c|c|c|c|c|c|c|}
\hline \multirow{2}{*}{ No. } & \multirow{2}{*}{ System } & \multirow{2}{*}{ Fleet deployment } & \multicolumn{15}{|c|}{ Station ID } \\
\hline & & & 1 & 2 & 3 & 4 & 5 & 6 & 7 & 8 & 9 & 10 & 11 & 12 & 13 & 14 & 15 \\
\hline \multirow[b]{2}{*}{1} & BSS + & Bikes & 48 & 0 & 33 & 40 & 42 & 37 & 50 & 29 & 21 & 40 & 42 & 44 & 27 & 21 & 26 \\
\hline & EBSS & E-bikes & 39 & 45 & 0 & 24 & 34 & 23 & 50 & 27 & 50 & 50 & 50 & 40 & 30 & 0 & 38 \\
\hline 2 & BSS & Bikes & 100 & 0 & 47 & 79 & 92 & 100 & 100 & 61 & 26 & 76 & 100 & 100 & 60 & 26 & 33 \\
\hline 3 & BSS & Bikes & 44 & 0 & 34 & 37 & 48 & 37 & 33 & 7 & 13 & 45 & 43 & 44 & 42 & 26 & 47 \\
\hline
\end{tabular}

beginning of planning horizon is bigger than or equal to that in system 3. This can be explained by the fact that the capacity of each station in system 3 is only half of that in system 2 , such that the optimal fleet deployment strategy at the beginning of the planning horizon is restricted by the capacity constraint.

The earned profits of systems 1-3 are USD 12223, 6508, and 3254, respectively, based on the current parameter setting (as shown in Table 3), and the results demonstrate the improvement in the earned profit of bike-sharing operators by introducing the shared e-bikes in the system. There are three components in the objective function (equation (8)) in the upper-level model, which represent the total revenue, the total holding cost, and the charging cost of e-bikes, respectively. The values of these revenue and cost components are also shown in Table 3. The results show that the bimodal integrated bike-sharing and e-bike-sharing system (system 1) can improve the total earned profit, which can mainly be explained by higher rental fee of e-bike compared to the traditional bike. Although the holding cost of traditional bike is lower than that of the e-bike and the charging cost can be incurred for e-bikes, the overall earned profit of system 1 is shown to be the highest.

5.3. The Effect of Shared E-Bike Fleet Size. The impact of the shared e-bike fleet size on the BSS has also been analysed, as shown in Figure 1. In the bike-sharing market, due to the oversupply and indiscriminate parking problem, governments are trying to control the fleet size or fleet utilisation rate in the market such that the balance between supply and demand can be kept. In countries like Singapore, the average utilisation rate for the entire shared bicycle is much lower than that of other cities like New York City and Chicago, where each bicycle is used three to six times a day [47]. The total fleet utilisation rate $u$ (trips per bike per day) of the integrated bike and e-bike-sharing system is calculated as

$$
u=\frac{\underset{e}{E}\left[\sum_{i} \sum_{j} \sum_{t}\left(x_{i j}^{e}(t)+\widehat{x}_{i j}^{e}(t)\right)\right]}{E_{e}\left[\sum_{i}\left(v_{i}^{e}(1)+\widehat{v}_{i}^{e}(1)\right)\right]} .
$$

Figure 1 depicts that the expected earned profit increases when the number of shared e-bikes added to the BSS increases, under the optimal fleet deployment plan. The fleet utilisation rate also increases when shared e-bikes are added (less than 50 shared e-bikes), as the travel time of e-bicyclists is shorter in comparison with traditional bicyclists for the same distance. However, the fleet utilisation rate of the whole system decreases by 0.04 trips/day as the number of shared e-bikes in the system increases from 50 to 700, which
TABLE 3: Revenue and cost.

\begin{tabular}{lccccc}
\hline No. & System & $\begin{array}{c}\text { Earned } \\
\text { profit }\end{array}$ & Revenue & $\begin{array}{c}\text { Holding } \\
\text { cost }\end{array}$ & $\begin{array}{c}\text { Charging } \\
\text { cost }\end{array}$ \\
\hline 1 & BSS + EBSS & 12223 & 12846 & 606 & 17 \\
2 & BSS & 6508 & 7040 & 532 & 0 \\
3 & BSS & 3254 & 3520 & 266 & 0 \\
\hline
\end{tabular}

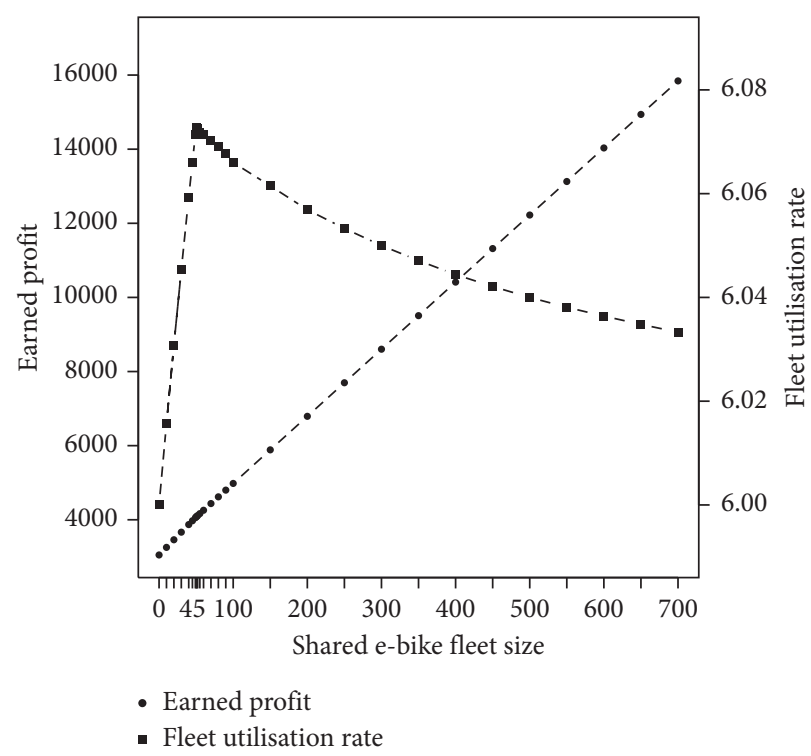

FIgURE 1: The effect of e-bike fleet size.

can be explained by the increment in e-bike supply under fixed demand level.

5.4. The Effect of Charging Time. The effect of charging time on the fleet deployment strategy is summarised in Table 4. As the charging time increases, both the earned profit and the fleet utilisation rate of the integrated bike-sharing and e-bike-sharing system decrease. The charging time directly impacts the number of e-bikes available at each e-bike station, and the results demonstrate the importance of e-bike charging efficiency to the performance of the whole system. The charging process of EBSS has also been addressed in the literature; for example, Thomas et al. [48] proposed a design framework for EBSS with regard to system components and user mobility patterns.

5.5. The Effect of E-Bike Pricing. The shared e-bike rental fee has direct impact on the generalised travel cost of e-bike 
TABLE 4: The effect of charging time.

\begin{tabular}{lcc}
\hline Charging time & Earned profit & Fleet utilisation rate \\
\hline Less than 2 hours & 12223 & 6.04 \\
2-4 hours & 7038.6 & 4.35 \\
$4-6$ hours & 5840.6 & 3.95 \\
\hline
\end{tabular}

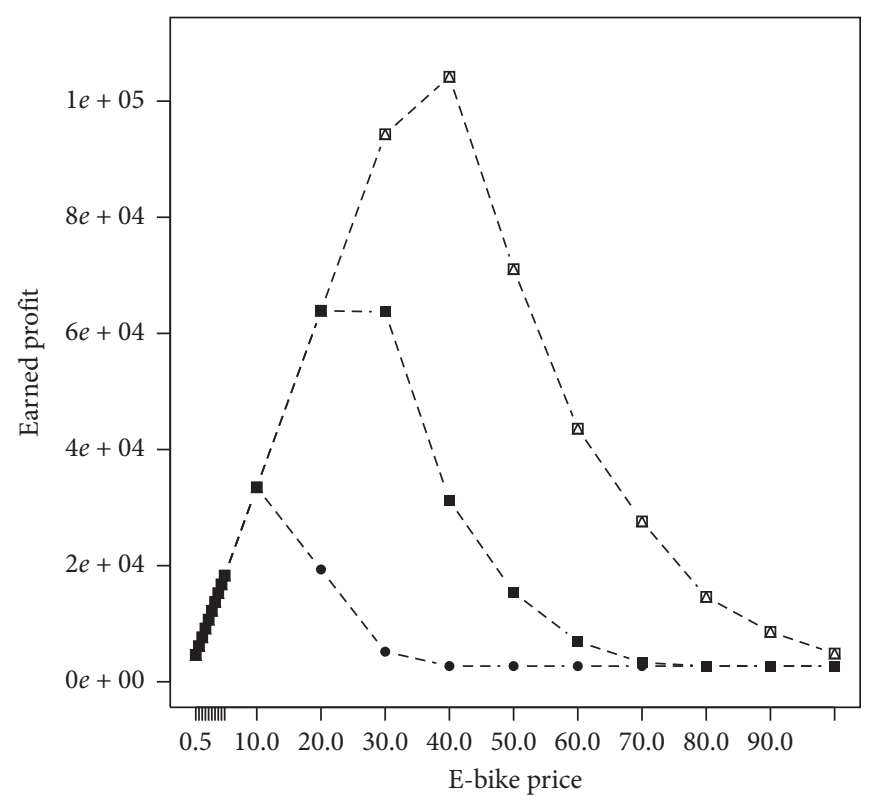

- Value of time $=5$

- Value of time $=10$

ه Value of time $=15$

Figure 2: Total earned profit.

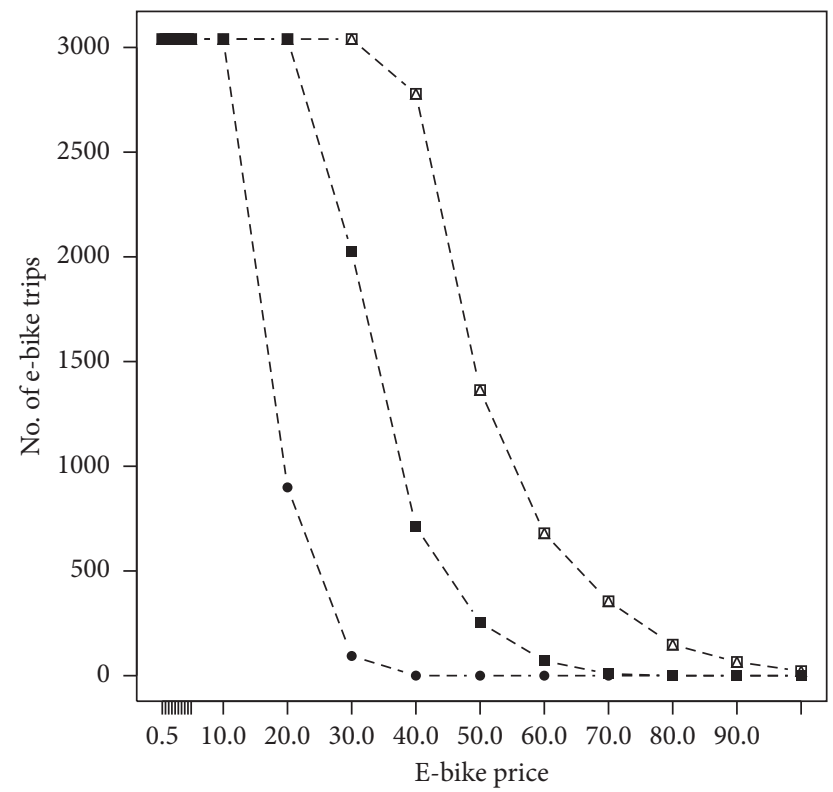

- Value of time $=5$

- Value of time $=10$

$\triangle$ Value of time $=15$

FIgURE 3: No. of e-bike trips. 
users, thereby affecting the traffic assignment between BSS and EBSS. The effect of e-bike pricing on the integrated bike and e-bike-sharing system is demonstrated in Figures 2 and 3. Figure 2 shows the effect of e-bike pricing on the total earned profit. At the beginning, the earned profit of EBSS increases linearly as the rental fee of shared e-bike increases, which can be explained by the constant number of e-bike trips completed in Figure 3. The low rental fee of e-bikes leads to relatively low generalised travel cost of e-bikes, such that the demand for EBSS is relatively high due to the modal split function (equation (26)). The demand for shared e-bikes exceeds the supply in this period, such that the number of satisfied e-bike trips remains constant. Thereby, the total earned profit increases as the price of shared e-bikes increases.

As the price of shared e-bike continues to increase, the demand for e-bike reduces under the multimodal user equilibrium condition, such that the number of e-bike trips also reduces correspondingly. In the end, the demand for EBSS will be constant at zero as the shared e-bike price grows, and the value of total earned profit is constant at the earned profit of BSS only minus the holding cost of e-bikes.

Figures 2 and 3 also demonstrate the effect of the value of time of travelers. In Figure 2, the critical point of e-bike price moves to the right as the value of time $\eta$ increases. Figure 3 also demonstrates that the number of satisfied e-bike trips is more sensitive to the e-bike price, when the value of time is lower. This can be explained by the fact that the travelers take the increment in monetary cost, that is, the shared e-bike price, as less important, as the value of time increases. Note that, for real-world application of the model and analysis, the value of time of various travelers should also be calibrated with trip survey data.

\section{Conclusions}

Following BSS, the shared e-bikes are becoming increasingly popular due to higher speed, reduced fatigue level, and so forth. However, the impact of shared e-bikes on the existing BSS has rarely been studied. This paper investigated the effect of shared e-bikes on bike-sharing system and determined the optimal fleet deployment strategy of both shared traditional bikes and shared e-bikes at each station. A stochastic multiperiod model is formulated to maximise the total earned profit of the integrated shared bike/e-bike system operator, and the branch-and-bound algorithm is applied to solve the integer linear programming problem. A numerical example is applied to test the validity of the model and the effectiveness of solution algorithm.

Some policy implications are summarised as follows: (1) when the fleet size is the same, shared e-bikes can provide higher earned profit in comparison with shared bikes. (2) As the fleet size of shared e-bikes added to the BSS increases, the earned profit increases, while the total fleet utilisation does not always increase. (3) As the charging time of shared e-bikes increases, the earned profit of system operator and fleet utilisation rate both decrease, demonstrating the significance of charging efficiency. (4) The pricing strategy of EBSS should be adjusted to achieve the optimal overall system performance. It is also necessary to calibrate the value of time of various travelers with trip survey data. However, there are still several limitations in the paper: For the numerical example, the demand between each pair of bike/e-bike stations is assumed to be known a priori. However, when the EBSS is newly introduced, different travel demand pattern could be demonstrated in reality. Real-world survey data should be collected and utilised to make the numerical example more realistic in future research. In addition, the proposed solution method has only been tested on a 15-station system whose size is small. In the future research, to solve larger size problems in real world, the proposed heuristic method could be adjusted with regard to the branching method, the termination criteria, and so forth, in order to maintain reasonable computation time. For future research, multimodal transportation environment can also be considered to cater for the traffic assignment among automobiles, buses, bicycles, e-bikes, and so forth. To model the problem, real-world trip data can also be applied if available. The model can also be improved by adding the determination of the optimal charging station number and location. Dynamic pricing system can also be investigated in future research to optimise the operation of the integrated bike and e-bike sharing system.

\section{Data Availability}

No data were used to support this study.

\section{Conflicts of Interest}

There are no conflicts of interest to declare.

\section{References}

[1] N.-F. Galatoulas, K. N. Genikomsakis, and C. S. Ioakimidis, "Spatio-temporal trends of e-bike sharing system deployment: a review in europe, north America and asia," Sustainability, vol. 12, no. 11, p. 4611, 2020.

[2] P. Zhong, A. Xu, Y. Chen, F. Gao, and G. Duan, "An optimization deployment scheme for static charging piles based on dynamic of shared e-bikes," in Proceedings 2019 15th International Conference on Mobile Ad-Hoc and Sensor Networks, pp. 343-347, Shenzhen, China, 2019.

[3] Z. Chen, Y. Hu, J. Li, and X. Wu, "Optimal deployment of electric bicycle sharing stations: model formulation and solution technique," Networks and Spatial Economics, vol. 20, no. 1, pp. 99-136, 2020.

[4] C. R. Cherry, H. Yang, L. R. Jones, and M. He, "Dynamics of electric bike ownership and use in kunming, China," Transport Policy, vol. 45, pp. 127-135, 2016.

[5] B. C. Langford, C. Cherry, T. Yoon, S. Worley, and D. Smith, "North America's first E-bikeshare," Transportation Research Record: Journal of the Transportation Research Board, vol. 2387, no. 1, pp. 120-128, 2013.

[6] Y. He, Z. Song, Z. Liu, and N. N. Sze, "Factors influencing electric bike share ridership: analysis of park city, Utah," Transportation Research Record: Journal of the Transportation Research Board, vol. 2673, no. 5, pp. 12-22, 2019.

[7] R. Dillet, "Bolt launches electric bike-sharing service in paris," 2020, https://techcrunch.com/2020/07/01/bolt-launcheselectric-bike-sharing-service-in-paris/.Online. 
[8] L. Laursen, "Copenhagen pioneers smart electric-bike sharing," 2013, https://spectrum.ieee.org/transportation/ alternative-transportation/copenhagen-pioneers-smartelectricbike-sharing.Online.

[9] R. Peace, "E-bike share schemes rolling out across UK," 2016, https://www.bike-eu.com/home/nieuws/2016/06/e-bikeshare-schemes-rolling-out-across-uk-10126690.Online.

[10] BikeMi, "What's BikeMi," 2020, https://www.bikemi.com/en/ service-info/info/whats-bikemi.aspx.Online.

[11] R. Liao, "Hellobike, survivor of china's bike-sharing craze, goes electric," 2019, https://techcrunch.com/2019/07/14/ china-micromobility-hellobike/.

[12] G. Celik, "Forget bike-sharing, e-bikes to become the next trend in china's sharing economy," 2020, https://kr-asia.com/ forget-bike-sharing-e-bikes-to-become-the-next-trend-inchinas-sharing-economy.Online.

[13] E. Fishman and C. Cherry, "E-bikes in the mainstream: reviewing a decade of research," Transport Reviews, vol. 36, no. 1, pp. 72-91, 2016.

[14] N. Popovich, E. Gordon, Z. Shao, Y. Xing, Y. Wang, and S. Handy, "Experiences of electric bicycle users in the sacramento, California area," Travel Behaviour and Society, vol. 1, no. 2, pp. 37-44, 2014.

[15] J. MacArthur, J. Dill, and M. Person, "Electric bikes in North America," Transportation Research Record: Journal of the Transportation Research Board, vol. 2468, no. 1, pp. 123-130, 2014.

[16] Z. Ling, C. Cherry, J. MacArthur, and J. Weinert, "Differences of cycling experiences and perceptions between e-bike and bicycle users in the United States," Sustainability, vol. 9, no. 9, p. 1662, 2017.

[17] J. Van Cauwenberg, I. De Bourdeaudhuij, P. Clarys, B. de Geus, and B. Deforche, "E-bikes among older adults: benefits, disadvantages, usage and crash characteristics," Transportation, vol. 46, no. 6, pp. 2151-2172, 2019.

[18] K. Wild and A. Woodward, "Why are cyclists the happiest commuters? health, pleasure and the e-bike," Journal of Transport \& Health, vol. 14, Article ID 100569, 2019.

[19] C. Cherry and R. Cervero, "Use characteristics and mode choice behavior of electric bike users in China," Transport Policy, vol. 14, no. 3, pp. 247-257, 2007.

[20] A. Fyhri and N. Fearnley, "Effects of e-bikes on bicycle use and mode share," Transportation Research Part D: Transport and Environment, vol. 36, pp. 45-52, 2015.

[21] I. Otero, M. J. Nieuwenhuijsen, and D. Rojas-Rueda, "Health impacts of bike sharing systems in europe," Environment International, vol. 115, pp. 387-394, 2018.

[22] S. Ji, C. R. Cherry, M. J. Bechle, J. D. Marshall, and J. D. Marshall, "Electric vehicles in China: emissions and health impacts," Environmental Science \& Technology, vol. 46, no. 4, pp. 2018-2024, 2012.

[23] G. Rose, "E-bikes and urban transportation: emerging issues and unresolved questions," Transportation, vol. 39, no. 1, pp. 81-96, 2012.

[24] R. Nocerino, A. Colorni, F. Lia, and A. Luè, "E-bikes and e-scooters for smart logistics: environmental and economic sustainability in pro-e-bike Italian pilots," Transportation Research Procedia, vol. 14, pp. 2362-2371, 2016.

[25] T. Weber, G. Scaramuzza, and K.-U. Schmitt, "Evaluation of e-bike accidents in Switzerland," Accident Analysis \& Prevention, vol. 73, pp. 47-52, 2014.

[26] S. Haustein and M. Møller, "E-bike safety: individual-level factors and incident characteristics," Journal of Transport \& Health, vol. 3, no. 3, pp. 386-394, 2016.
[27] B. C. Langford, J. Chen, and C. R. Cherry, "Risky riding: naturalistic methods comparing safety behavior from conventional bicycle riders and electric bike riders," Accident Analysis \& Prevention, vol. 82, pp. 220-226, 2015.

[28] Y. Guo, J. Zhou, Y. Wu, and J. Chen, "Evaluation of factors affecting e-bike involved crash and e-bike license plate use in China using a bivariate probit model," Journal of Advanced Transportation, vol. 2017, Article ID 2142659, 12 pages, 2017.

[29] F. Hu, D. Lv, J. Zhu, and J. Fang, "Related risk factors for injury severity of e-bike and bicycle crashes in hefei," Traffic Injury Prevention, vol. 15, no. 3, pp. 319-323, 2014.

[30] C. Cherry, S. Worley, and D. Jordan, "Electric bike sharing-system requirements and operational concepts," Technical Report, University of Tennessee, Knoxville, TN, USA, 2010.

[31] V. Manzoni, F. Codecà, A. Moia, and S. M. Savaresi, "Integration of electric pedal assisted bikes in a bike-sharing system," IFAC Proceedings Volumes, vol. 42, no. 15, pp. 174-181, 2009.

[32] A. A. Campbell, C. R. Cherry, M. S. Ryerson, and X. Yang, "Factors influencing the choice of shared bicycles and shared electric bikes in beijing," Transportation Research Part C: Emerging Technologies, vol. 67, pp. 399-414, 2016.

[33] S. Ji, C. R. Cherry, L. D. Han, and D. A. Jordan, "Electric bike sharing: simulation of user demand and system availability," Journal of Cleaner Production, vol. 85, pp. 250-257, 2014.

[34] C. Li, Z. Dai, W. Peng, and J. Shen, "Green travel mode: trajectory data cleansing method for shared electric bicycles," Sustainability, vol. 11, no. 5, p. 1429, 2019.

[35] Y. Hu, Z. Chen, and X. Wu, "Station allocation model for electric bicycle-sharing system," Technical Report, 2017.

[36] L. M. Martinez, L. Caetano, T. Eiró, and F. Cruz, “An optimisation algorithm to establish the location of stations of a mixed fleet biking system: an application to the city of lisbon," Procedia - Social and Behavioral Sciences, vol. 54, pp. 513-524, 2012.

[37] H. Sayarshad, S. Tavassoli, and F. Zhao, "A multi-periodic optimization formulation for bike planning and bike utilization," Applied Mathematical Modelling, vol. 36, no. 10, pp. 4944-4951, 2012.

[38] J.-R. Lin, T.-H. Yang, and Y.-C. Chang, "A hub location inventory model for bicycle sharing system design: formulation and solution," Computers \& Industrial Engineering, vol. 65, no. 1, pp. 77-86, 2013.

[39] C.-C. Lu, "Robust multi-period fleet allocation models for bike-sharing systems," Networks and Spatial Economics, vol. 16, no. 1, pp. 61-82, 2016.

[40] S. Yan, C.-C. Lu, and M.-H. Wang, "Stochastic fleet deployment models for public bicycle rental systems," International Journal of Sustainable Transportation, vol. 12, no. 1, pp. 39-52, 2018.

[41] S. Zhu, "Stochastic bi-objective optimisation formulation for bike-sharing system fleet deployment," Proceedings of the Institution of Civil Engineers - Transport, vol. 1, 2020.

[42] L. Caggiani, R. Camporeale, M. Marinelli, and M. Ottomanelli, "User satisfaction based model for resource allocation in bike-sharing systems," Transport Policy, vol. 80, pp. 117-126, 2018.

[43] Z.-C. Li, M.-Z. Yao, W. H. K. Lam, A. Sumalee, and K. Choi, "Modeling the effects of public bicycle schemes in a congested multi-modal road network," International Journal of Sustainable Transportation, vol. 9, no. 4, pp. 282-297, 2015.

[44] L. Zhang, H. Yang, D. Wu, and D. Wang, "Solving a discrete multimodal transportation network design problem," 
Transportation Research Part C: Emerging Technologies, vol. 49, pp. 73-86, 2014.

[45] J. Nocedal and S. Wright, "The dual simplex method," in Numerical Optimization, pp. 382-385, Springer Science \& Business Media, New York, NY, USA, 2006.

[46] MATLAB, "Mixed-integer linear programming algorithms," 2020, https://www.mathworks.com/help/optim/ug/mixedinteger-linear-programming-algorithms.html.

[47] The Straits Times, "Brakes applied-shared bike fleets to plunge," 2018, https://www.straitstimes.com/singapore/ transport/brakes-applied-shared-bike-fleets-to-plunge.

[48] D. Thomas, V. Klonari, F. Vallée, and C. S. Ioakimidis, "Implementation of an e-bike sharing system: the effect on low voltage network using pv and smart charging stations," in Proceedings of the 2015 International Conference on Renewable Energy Research and Applications (ICRERA), pp. 572-577, IEEE, Ankara, Turkey, 2015. 